Filigrane

Écoutes psychanalytiques

\title{
Deux courts textes métapsychologiques
}

\section{Dominique Scarfone}

Volume 28, numéro 1, 2019

URI : https://id.erudit.org/iderudit/1064599ar

DOI : https://doi.org/10.7202/1064599ar

Aller au sommaire du numéro

\section{Éditeur(s)}

Santé mentale et société

\section{ISSN}

1192-1412 (imprimé)

1911-4656 (numérique)

Découvrir la revue

Citer cet article

Scarfone, D. (2019). Deux courts textes métapsychologiques. Filigrane, 28(1),

91-101. https://doi.org/10.7202/1064599ar d'utilisation que vous pouvez consulter en ligne.

https://apropos.erudit.org/fr/usagers/politique-dutilisation/ 


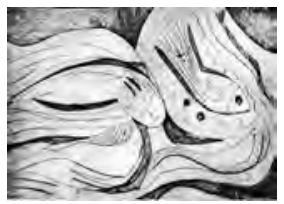

\section{Deux courts textes métapsychologiques ${ }^{1}$}

\section{Dominique Scarfone}

\section{L'inconscient qui parle et l'inconscient dont on parle ${ }^{2}$}

E n 1923-1924, dans «Le moi et le ça» - son dernier grand texte méta- psychologique - Freud remet en question le sens systémique qu'il avait donné au terme "inconscient». Puisqu'une grande partie du moi doit désormais être considérée inconsciente, l'opposition cs/ics ne lui apparait plus aussi utile. Le sens qualitatif du mot « inconscient» reprend le dessus et Freud se sent obligé de "concéder que le caractère d'être inconscient perd pour nous en significativité. Il se change en une qualité multivoque...» (Freud, 1923, p. 263) Toutefois, il ne peut s'empêcher de noter que «la propriété consciente ou non est l'unique flambeau dans l'obscurité de la psychologie des profondeurs» (ibid.). Comment résoudre cet apparent dilemme?

La voie qu'emprunte aussitôt Freud, dans le même texte, est de s'intéresser non plus à l'état conscient ou inconscient, mais au devenir conscient, c'est-à-dire au mouvement entre ics et cs. Ce devenir conscient exige, écrit-il, de faire transiter les contenus inconscients par les canaux de la perception du monde extérieur, et c'est ce que permet la parole en analyse. Il me semble ouvrir par là, implicitement, le chemin vers une définition opérationnelle de l'inconscient dans sa différence avec le préconscient-conscient.

L'inconscient se distingue désormais par l'absence des qualités qui sont le propre du conscient. Il faut donc se demander ce que ces qualités permettent (ou ce que leur absence empêche). Ainsi posé, l'être conscient ou inconscient n'est plus une question de présence ou d'absence dans le champ perceptif, mais renvoie à une fonction que l'appareil psychique remplit à travers le devenir conscient. En effet, si le devenir conscient demande de passer par la perception, celle-ci n'est pas une fin en soi: elle met «à portée de main ", c'est-à-dire qu'elle rend propre à un certain usage ce qui jusque-là était inaccessible. Rendre propre à un certain usage, c'est donner un sens, si, comme l'a écrit Wittgenstein (1953), le sens c'est l'usage. Nous dirons qu'est conscient ce qui possède certaines qualités permettant de donner un sens ou un usage, réel ou potentiel. 
Mais quel sens? Et un sens donné à quoi?

Je propose de distinguer entre un inconscient non-structuré, qui se pose comme question, comme problème ou énigme, et un inconscient structuré, constitué d'un ensemble de réponses construites au cours du temps, mais qui œuvrent désormais à l'insu du sujet, organisant sa vie fantasmatique.

L'inconscient comme question, non structuré, c'est la Chose inconsciente, cette énigme qui persiste dans la rencontre de l'autre humain et que Freud a repérée dès le Projet de 1895. De cette Chose (Ding), sur laquelle Lacan (1959-1960) a attiré l'attention, je retiendrai qu'elle est par définition non-symbolisée; elle n'est donc pas insérée dans une structure, sauf comme béance, trou noir au centre de la galaxie psychique. L'inconscient-question, c'est le refoulé originaire, reste énigmatique du message venu de l'autre et contaminé par le Sexual (Laplanche, 2006); refoulé parce que résistant à la traduction (Freud, 1896). L'inconscient-question est donc par essence sexuel. Par conséquent, parler d'un inconscient "structuré» (comme un langage ou autrement), c'est se référer non à cette Chose, mais à un inconscient où sont repérables des formations agencées en structures qui «habillent» la Chose sexuelle (Scarfone, 2014). Les formations de cet inconscient-réponse résultent des tentatives de résoudre l'énigme de l'autre (pensons par exemple aux théories sexuelles infantiles); des réponses formulées avec les instruments mytho-symboliques que fournit ou impose la culture, tout d'abord à travers la sous-culture familiale (Castoriadis-Aulagnier, 1975).

La face de l'inconscient qui est repérable d'ordinaire par l'analyse, ce sont donc les structures résultant des effets combinés de la Chose inconsciente et des formes proposées par la culture. Ces agencements inconscients se reconnaissent par les effets perturbateurs de la Chose (rêves, lapsus, actes manqués, etc.). Ils ne sont pas une manifestation directe de la Chose, mais les indices de sa «force d'attraction» (Pontalis, 1990) qui dévie le cours «normal» des processus psychiques. Ce cours «normal» n'est évidemment nulle part repérable puisque tout humain est habité par la Chose inconsciente, quel que soit son fonctionnement psychique. Il n'y a de normalité que comme asymptote ou comme ligne médiane idéale (voire idéologique), chaque culture développant la sienne.

Les formations psychiques inconscientes produites comme réponses ou habillages sont toujours déjà déformées, perturbées par la Chose: symptômes, avec leurs fantasmes sous-jacents; identifications, délires, etc. À strictement parler, cet «inconscient structuré» appartient, selon la première topique, au préconscient, celui-ci pouvant toutefois être fortement polarisé 
par l'attraction de la Chose au point de se présenter comme totalement étranger, «en forme de ça».

L'analyse, la déconstruction, la détraduction de ces formations laissera éventuellement apparaitre l'altérité radicale, l'Unheimliche - effet plus senti de la Chose quand fait défaut l'habillage préconscient. Cela donne des moments de désymbolisation, de désidentification, allant jusqu’à la dépersonnalisation en cours d'analyse. Devant l'indicible de la Chose, le transfert prend le relais. Transferts «en plein» ou «en creux» (Laplanche, 1991), ces nouvelles expériences de l'exposition à l'énigme de l'autre - ici incarné par l'analyste - permettront, dans le cadre de l'analyse, de nouvelles traductions ou symbolisations. Celles-ci seront la face structurante (l'ics-réponse) de nouveaux refoulements, puisque toute traduction est aussi refoulante et que la Chose (l'ics-question) persiste, jamais totalement traduite ou symbolisée.

On a donc un inconscient qui parle, qui fait des tentatives de réponse à un inconscient dont on parle, qui, lui, est une question qui se pose sans fin.

\section{Une psychanalyse en mouvement ${ }^{3}$}

La question «Qu'est-ce qu'on ne sait pas encore en psychanalyse?» fait impression. Elle nous somme implicitement de faire d'abord l'inventaire de tout ce que nous savons, ou croyons savoir, pour en déduire ensuite ce qui resterait à découvrir. Cela semble tout à fait normal dans les sciences naturelles, même si là aussi il est dangereux de chercher à établir ce que l'on ne sait pas encore. Vers la fin du XIX ${ }^{e}$ siècle, Lord Kelvin avait cru pouvoir affirmer que la physique avait découvert tout ce qu'il y avait à savoir, et qu'il ne restait plus qu'à faire des mesures plus précises... Quelques années plus tard vinrent Planck et Einstein... Ferions-nous la même erreur en psychanalyse? Freud lui-même avait failli la faire lorsque, en 1915-17, ayant écrit quelques-uns des douze chapitres projetés de sa Métapsychologie, il pensait avoir dit tout ce qu'il avait à dire sur le sujet. Puis vint $A u$-delà $d u$ principe de plaisir...

On ne peut certes aujourd'hui se résoudre à penser qu'il n'y a plus rien de nouveau à introduire en psychanalyse. Mais pouvons-nous pour autant tracer, ne serait-ce qu'en négatif, la figure de ce que nous ignorerions? À moins que la formulation elle-même de la question ne soit pas pertinente dans notre domaine? Quoi qu'il en soit, même en l'acceptant telle quelle, la question semble porteuse d'importantes difficultés, qui tiennent d'une part au problème de comment inventorier ce que nous savons, mais plus encore au problème du rapport de la psychanalyse au savoir en tant que tel. 
Notons pour commencer qu'il y a une ironie, sans doute involontaire, à se demander ce qu'on ne sait pas en psychanalyse, si l'on songe que l'insu (Un-bewusste) est précisément son objet spécifique. Bien que la proposition ne soit pas tout à fait exacte, on peut dire qu'avant Freud, l'homme avait un inconscient mais ne le savait pas: l'inconscience, donc, était double. Depuis Freud, la deuxième inconscience (ne pas savoir que l'on a un inconscient) pourrait sembler levée. Mais est-ce vraiment le cas? Si les psychanalystes ont appris de Freud à voir et à entendre l'inconscient se manifester, s'ils savent en capter les incidences, peuvent-ils pour autant savoir ce qu'est l'inconscient? Je crois qu'il est permis d'en douter. Nous en parlons comme si nous savions positivement ce que c'est. Il vaut sans doute la peine de considérer que le mot «inconscient» renvoie à au moins deux niveaux de fonctionnement. Dans la section précédente, j'ai proposé de distinguer l'inconscient «qui parle» de l'inconscient «dont on parle». L'inconscient qui parle, c'est-à-dire un inconscient "structuré», constitué de réponses, et un inconscient "non structuré», conçu comme question. Lorsque nous croyons savoir ce qu'est l'inconscient, nous nous référons probablement à l'inconscient "qui parle", celui dont Freud (1915) disait que lorsque nous l'abordons «selon un mode de considération [...] procédant de la conscience [...] toute la somme des processus psychiques vient se poser comme le royaume du préconscient» (p. 232). Le royaume du préconscient peut en effet nous paraître comme un espace ou une entité positive, avec des contenus psychiques. N'oublions pas, cependant, que son caractère inconscient nécessite l'hypothèse d'un inconscient radical, celui dont les effets ont conduit à la formation de compromis entre la poussée inconsciente et les unités mytho-symboliques fournies par la culture; un inconscient qui ne parle pas mais dont nous sommes bien forcés de parler.

On peut craindre toutefois qu'à force de parler de cet inconscient au sens plus strict, on n'oublie ou à tout le moins néglige sa radicale étrangeté. Au fait, le choix de pratiquer la psychanalyse ne serait-il pas au fond un effort visant à une certaine "saisie», à une "com-préhension» de l'inconscient? Familiarisé avec les écrits psychanalytiques, on croit savoir, au moins intellectuellement, qu'il y a de l'inconscient. Les difficultés commencent quand nous essayons d'en dire quelque chose. Nous voilà alors dans un champ de mines méthodologiques et épistémologiques. C'est que la tentation est grande de dire sur un mode positif ce que nous savons ou croyons savoir, de 
développer une ontologie de l'inconscient. Or il n'est pas sûr que ce soit la bonne façon de s'y prendre.

Le problème est de voir si nous pouvons détacher, distinguer "positivement» un "quoi» inconscient de son «comment», de ses incidences. En d'autres mots, avons-nous affaire à une entité qui se nomme inconscient ou bien à un ensemble de processus - un mode de fonctionnement psychique dont les turbulences nous obligent à postuler un inconscient? L'inconscient se laisserait-il mieux saisir dans sa "choséité» s'il était conçu comme entité, comme fait positif plutôt que comme un ensemble de processus ou un faisceau de mouvements? Je crois bien que personne ne cherche à situer l'inconscient dans une région particulière du cerveau, mais avons-nous vraiment pris Freud au sérieux lorsqu'il a souligné que l'appareil psychique est une «fiction», ou que «les pulsions sont des êtres mythiques»? À l'opposé, si la psyché n'est que mouvements, processus, comment alors penser la fixation, la stase libidinale, les «contenus» inconscients et leur «contenant»?

Pour qui s'inquiéterait d'une "perte de substance» de l'inconscient conçu comme processus, on peut aisément concevoir qu'une manière différente de fonctionner, une inflexion particulière du cours des événements psychiques, peut présenter le même effet de réalité qu'une structure ou qu'un «contenu» inconscient. À l'opposé, quand nous en parlons comme d'une entité, nous savons que nous utilisons une métaphore puisque personne ne peut «voir» ou «toucher» l'inconscient. Le problème est que même si nous utilisons sciemment un langage métaphorique, il n'est pas sûr que nous échappons aux pièges de la métaphore elle-même. Ainsi, ne sommes-nous pas portés à poser des fantasmes comme existant «dans» l'inconscient?

Pour ma part, je propose de retenir de Freud la leçon du «comment» plutôt que celle du «quoi». Comment (avec quelle méthode et quelle éthique) approcher le problème de l'inconscient; comment (selon quels processus) ça semble marcher; comment (sous quels aspects) ça se manifeste. Ces trois comment, la clinique guidée par l'hypothèse d'un inconscient effectif (wirklich) peut aisément nous les indiquer: associations libres, disposition à écouter avec une attention en égal suspens, processus primaires, irruption intempestive, formation de compromis (rêves et symptômes, lapsus, trous de mémoire temporaires, délire), etc. L'inconscient se manifeste alors par sa capacité de perturber le cours des événements psychiques, donc par un effet déstabilisant plutôt que par des contours positifs. Nous assistons au dérangement d'un cours d'événements, cours dont nous ne pouvons que construire l'allure idéale, non perturbée, puisque nous ne savons pas - ni 
ne pouvons savoir - ce que serait un cours des événements psychiques non affecté par l'inconscient.

Poser l'inconscient en termes de processus n'enlève rien à sa réalité et à son effectivité (Wirklichkeit). Des conséquences différentes peuvent découler d'un rapport différent entre des processus sans avoir pour autant à postuler des entités définies spatialement. Certes, on peut comme Aulagnier (1975) parler d'un «espace où le Je peut advenir»; il reste que pour elle «le Je est un savoir du Je sur le Je» (p. 169). Parler d'espace est sans doute, dans ce cas, une concession inévitable au langage courant; après tout, cinq cents ans après Copernic, nous parlons toujours d'un «beau coucher de soleil» - et pourquoi pas? Tant que nous ne demandons pas aux astrophysiciens de nous confirmer que c'est bien le soleil qui «se couche», l'expression ne fait de mal à personne. Cependant, dans une conception rigoureuse, on n'a nul besoin d'hypostasier les processus inconscients en entités discrètes. Si nous suivons cette idée jusqu'au bout, alors on peut dire que nous ne savons pas, et ne pouvons pas savoir, ce qu'est l'inconscient, tout simplement parce que l'inconscient... n'est pas. On peut nommer inconscient la manifestation à travers des effets particuliers, d'une forme particulière du mouvement psychique ou de ses précurseurs.

On pourrait toutefois craindre que le fait d'opter pour cette version «en mouvement» ou «en processus» soit l'amorce d'une tendance à réduire l'inconscient à sa version purement qualitative: l'inconscient ne serait qu'un mouvement psychique particulier, dépourvu du caractère conscient. Le pas suivant serait celui de ramener ce mode d'opérer à un fait de nature et de réduire le problème de l'inconscient à celui de mécanismes neutres dans le champ des rapports inter-humains. Cela renverrait à une version de l'inconscient cognitif. En caricaturant un peu, on pourrait imaginer un sujet déclarant quelque chose comme: «Pardonnez mon acte manqué, ce matin l'inconscient marche plus fort que d'habitude...» Évanouie la conflictualité et la résistance, évaporé le souhait inconscient, aboli l'Unheimliche. On perdrait ainsi une dimension essentielle de l'inconscient freudien, soit son incompatibilité avec le Je (ou le moi). Un Je qui est obligé de constater que «quelque chose» lui échappe, opère à son insu, mais un Je qui doit tout de même prendre acte d'une adresse, d'une incidence inévitable et problématique de ce «quelque chose» dans les rapports inter-humains. 
Dans Le Moi et le Ça, c'est-à-dire au moment même où Freud est tenté d'abandonner la notion d'inconscient systématique pour ne s'en tenir qu'à la qualité consciente (ou à son absence), il semble aussitôt amené à réagir à ce retour en arrière en posant l'inconscient comme un "autre-chose» (etwas anderes) (Freud, 1923, p. 266-267), c'est-à-dire quelque chose d'étranger et d'incompatible avec le moi, ce qui le conduira à adopter le terme de "ça». On semble alors, avec les mots «autre-chose» et «ça» être bel et bien dans le domaine des entités stables plutôt que des processus. Pourtant, plus loin dans le même texte, Freud comparera le moi et le ça en ces termes: «La perception joue pour le moi le rôle qui dans le ça échoit à la pulsion» (Freud, 1923, p. 269). Il est donc question de processus (perception, pulsion), ce qui se confirmera dans des textes ultérieurs, quand Freud dira des pulsions qu'elles sont «des êtres mythiques, grandioses dans leur indétermination » (Freud, 1932, p. 178). Nous voici donc devant très peu de substances: «fiction» d'un appareil psychique, pulsions, «êtres mythiques». Et pourtant, cela a des incidences bien réelles. Pouvons-nous rendre compte de ces incidences sans recourir au «quoi» et en n'examinant que le «comment»?

La pratique et sa théorisation nous apprennent que les effets de l'inconscient, ce sont des sortes d' "arrêts» dans la marche des processus psychiques. Arrêts qui ne signifient pas immobilité, puisque ce sont en fait des boucles de répétition: répétition dans les symptômes et répétition aussi dans la constitution même du moi. Les traits de caractère du moi, voilà un des premiers exemples de répétition que donne Freud (1914) dans son texte «Remémoration, répétition, perlaboration». Cela nous invite à poser que le moi lui-même est une sorte de processus répétitif, lui-même une hypostase, une formation qui semblerait se cristalliser pour de bon, si ce n'était qu'elle est constamment soumise au courant incessant des processus pulsionnels, courant et processus que ce même moi subit comme des assauts et contre lesquels il se défend. Il n'y a donc pas d'arrêt véritable (ce serait la mort), mais ce qui ainsi semble se fixer peut être conçu comme une forme se répétant à très haute vitesse. Dans ce sens, nous pouvons affirmer que le moi se constitue par refoulement, et même dire quelque chose de plus au sujet de ce refoulement. Le refoulement, que Freud considère comme un des piliers de la théorie, peut se décrire de bien des façons, mais ici nous pourrions le décrire comme précisément la façon qu’a le moi de se penser comme substance (Levinas, 1967), qui est aussi sa façon de résister au cours inéluctable des événements psychiques. Le moi est donc aussi essentiellement résistance. Ce refoulement, cette résistance, ne serait-ce pas le parcours, répété à 
très haute vitesse, des circuits «bien frayés» que Freud appelait «réseau de neurones» (Freud, 1895) et que nous pouvons concevoir comme réseau de représentations (Laplanche, 1970)? Si oui, s'impose aussitôt une remarque supplémentaire: les représentations sont elles-mêmes des manières de substantialiser des processus. Avec pour conséquence que l'inconscient ne peut être constitué de représentations, fussent-elles des «représentations de choses»(Sachvorstellungen), la représentation étant par excellence un produit de l'hypostase, une sorte d'arrêt sur image (freeze-frame). Pourtant, l'expérience même de l'analyse nous montre que nous ne pouvons échapper totalement aux effets de l'hypostase. Qu'une sorte de fétichisme indépassable opère dans la pensée, fétichisme découlant d'un effort de se saisir de l'inconscient. De sorte qu'il est difficile sinon impossible de savoir ce que serait un sujet humain exempt de cette tendance à l'hypostase, à la substantialisation.

Nous étant maintenant privés de représentations inconscientes, il nous faut bien tenter de donner une autre idée de ce qui meut l'inconscient. En bonne tradition freudienne, nous préférerons parler de «traces» plutôt que de représentations, mais cela ne fait que nous renvoyer au problème de concevoir la nature exacte de ces traces. Avec le mot «trace», nous sommes naturellement portés à nous en faire une image - des traces de pas dans le sable, par exemple -, et il est difficile d'aller bien au-delà de cette... représentation! On voit bien quel effort soutenu est nécessaire pour résister à la conception positive, «substantialiste», de l'inconscient et s'en tenir plutôt à des processus, à des mouvements.

\section{Savoir, savourer...}

Peut-être y a-t-il problème dans la formulation même de la question à laquelle nous tentons ici de répondre? Peut-être est-il erroné ou improductif de poser la question de la psychanalyse en tant que savoir?

Certes, il y a un savoir psychanalytique pouvant être enseigné et transmis. Mais il importe de ne jamais perdre de vue ce que savoir suppose: une position à distance, en détachement, en extériorité par rapport à l'objet de ce savoir. Cela entraîne une conception de la psychanalyse où la relation sujet-objet concernerait un objet extérieur et un sujet qui ne se laisserait pas affecter, altérer par l'expérience. Cette position serait, à la limite, admissible comme point de départ, avant que ne s'engage le transfert. Après tout, nous nous attendons à ce qu'un psychanalyste sache instituer et maintenir le cadre de la séance, qu'il ait un certain «know-how» et qu'il puisse aussi 
se repérer parmi un certain nombre de configurations cliniques. Mais ce savoir, s'il n'est pas lui-même mis en suspens, va vite devenir une résistance, voire la principale résistance, au mouvement de l'analyse. Ce mouvement exige, entraîne une mobilisation psychique différentielle des deux sujets engagés dans l'analyse et l'on peut même aller jusqu'à dire, comme Michel de M'Uzan avec la notion de Chimère, que la relation analytique constitue une sorte de créature nouvelle ayant des caractéristiques irréductibles à l'un ou l'autre des deux participants.

Quand s'engage le processus psychanalytique, notamment par le fait du transfert, il se constitue comme un système, un processus que l'on ne saurait observer de l'extérieur sans aussitôt le détruire ou le dénaturer. C'est dans ce sens qu'il ne peut être question de «savoir», sauf à penser ce terme à partir de sa racine étymologique latine sapere, qui concerne le fait de savourer. Or, si l'on peut, après coup, faire rapport à un tiers de l'expérience analytique, il n’y a que les deux sujets engagés dans l'expérience elle-même qui «savourent» et donc «savent» vraiment ce qui s'éprouve dans cette analyse particulière. Et comme celle-ci dépend de la rencontre de deux sujets uniques, elle est par définition non-reproductible et, par là, ne peut jamais se prêter à un savoir intégralement communicable.

Il y a des psychanalystes qui croient pouvoir aborder positivement la question du savoir psychanalytique en conjuguant psychanalyse et neurosciences. L'effort «neuro-psychanalytique» a peut-être le mérite de nous rappeler sans cesse l'assise corporelle, cérébrale - entre autres - de Psyché. Mais il faut en ce domaine se garder d'un écueil majeur, d'un présupposé implicite qui ferait s'équivaloir les mécanismes cérébraux et les mécanismes psychiques. À partir de là, en effet, la tentation est forte de ramener un déni, une projection, une formation réactionnelle, etc. à une erreur d'aiguillage, à un dysfonctionnement neuro-cognitif, à une anomalie neuronale. Je disais plus haut que nous ne saurons jamais ce que seraient des processus psychiques non perturbés par l'inconscient. À présent il convient de poser clairement que le refoulement, par exemple, n'est pas une erreur neuronale, ne correspond à aucune anomalie cérébrale. Le transfert non plus n'est pas une erreur d'attribution, un problème de traitement défectueux des perceptions. Le transfert est un processus, un fait universel survenant dans toute relation asymétrique; un fait que la psychanalyse a le mérite d'avoir mis en évidence et d'avoir su faire travailler au 
service d'une meilleure connaissance de soi et de l'autre ainsi que de la résolution de certaines impasses psychiques. Cela, il est certes possible et important de le savoir... Mais ce n'est qu'en s'engageant dans la parole en analyse que l'on «savourera» ce qu'il en est de ce transfert. La seule expérience non analytique qui donne ce savoir (au sens de sapere) est sans doute incarnée dans l'œuvre des poètes, écrivains et artistes qui, par leur art, réussissent à transmettre quelque chose qui se situe au-delà du savoir objectivant, et susciter en nous, lecteurs ou spectateurs, un transfert sur leurs œuvres.

Je dis en somme que le savoir positif acquis à partir - et au sujet - de la psychanalyse est toujours susceptible de nous faire perdre de vue l'irréductibilité de l'inconscient à ce savoir. De plus, cela risque de nous conduire sur la voie de la «technologisation» du travail clinique, ce qui correspondrait à une tout autre éthique que celle de la psychanalyse. En effet, le savoir positif n'a de valeur que dans la mesure où il permet une action, une intervention opérée de l'extérieur, une maîtrise technique dans une visée de pouvoir. Le savoir, soulignons-le, est recherché par l'entremise de la pulsion d'emprise (Bemächtigungstrieb). Et tout savoir comporte son propre refoulement de l'insu.

Qu'opposer à ce savoir-emprise? Je crois que Freud nous a légué pour cela un instrument précieux sous le nom de «métapsychologie». Ce terme est souvent conçu dans la littérature psychanalytique comme un ensemble de «théories spéculatives» élaborées par Freud dans certains de ses textes. Pour ma part, je conçois la métapsychologie non comme une formulation théorique, mais comme un mouvement toujours recommencé $d u$ penser freudien, le penser de Freud et celui de ceux qui reconnaissent que la méthode psychanalytique est avant tout une forme particulière, une tournure originale de la pensée quand celle-ci se laisse «aimanter» par les courants psychiques que nous nommons «inconscient».

À la question «Qu'est-ce qu'on ne sait pas encore en psychanalyse?», je suis tenté de répondre qu'on ne sait pas encore assez se méfier de la tentation du savoir positif, un savoir qui, comme suggéré plus haut, peut conduire à l'opposé de ce que visent la raison et la méthode freudiennes. 


\section{Notes}

1. Les courts articles qui suivent ont été produits tous deux à l'invitation du comité de rédaction de la revue Calibán que publie la Fédération latino-américaine de psychanalyse (FEPAL). Pour le premier, j'avais été invité à répondre à la question «De quel inconscient parlons-nous?»; pour le second, à la question "Qu'est qu'on ne sait pas encore en psychanalyse?»

2. Article paru sous le titre «El inconsciente que habla y el inconsciente del que hablamos», Calibán, Revista Latinoamericana de Psicoanálisis, 13/2 (2015), 137-139.

3. Article paru sous le titre "Un Psicoanálisis en movimiento», Calibán, Revista Latinoamericana de Psicoanálisis, 14/2 (2016), 14-20.

\section{Références}

Castoriadis-Aulagnier, P. (1975). La violence de l'interprétation. Paris: Presses universitaires de France.

De M’Uzan, M. (2015). L'inquiétude permanente. Paris: Gallimard.

Freud, S. (1895). Projet d'une psychologie. Dans Lettres à Wilhelm Fliess. Paris: Presses universitaires de France, 2006.

Freud, S. (1896). Lettre du 6 décembre 1896. Dans Lettres à Wilhelm Fliess., op. cit.

Freud, S. (1914). Remémoration, répétition, perlaboration. Dans Euvres complètes XII. Paris: Presses universitaires de France.

Freud, S. (1915). L'inconscient. Dans Euvres complètes XIII. Paris: Presses universitaires de France.

Freud, S. (1923). Le moi et le ça. Dans Euvres complètes XVI. Paris: Presses universitaires de France.

Freud, S. (1932). Angoisse et vie pulsionnelle. Dans Euvres complètes XIX. Paris: Presses universitaires de France.

Lacan, J. (1959-1960). Le Séminaire. Livre VII. L'éthique de la psychanalyse. Paris: Seuil, 1984. Laplanche, J. (1970). Vie et mort en psychanalyse. Paris: Flammarion.

Laplanche, J. (1991). Du transfert: sa provocation par l'analyste. Dans La primauté de l'autre en psychanalyse. Paris: Flammarion, 1997.

Laplanche, J. (2006). Sexual. La sexualité élargie au sens freudien. Paris: Presses universitaires de France.

Levinas, E. (1967). Ruine de la représentation. Dans En découvrant l'existence avec Husserl et Heidegger. Paris: Vrin.

Pontalis, J.-B. (1990). La force d'attraction. Paris: Seuil.

Scarfone, D. (2014). L'impassé, actualité de l'inconscient. Revue française de psychanalyse, 78 (5), 1357-1428.

Wittgenstein, L. (1953). Philosophical Investigations. London: Blackwell. 